\title{
Pericyte MyD88 and IRAK4 control inflammatory and fibrotic responses to tissue injury
}

\author{
Irina A. Leaf, ${ }^{1,2}$ Shunsaku Nakagawa, ${ }^{3}$ Bryce G. Johnson, ${ }^{1,3}$ Jin Joo Cha, ${ }^{3}$ Kristen Mittelsteadt, ${ }^{3}$ Kevin M. Guckian, ${ }^{1}$ Ivan G. Gomez, ${ }^{1,3}$ \\ William A. Altemeier, ${ }^{3}$ and Jeremy S. Duffield ${ }^{1,3}$
}

${ }^{1}$ Research and Development, Biogen, Cambridge, Massachusetts, USA. ${ }^{2}$ Center for Infectious Disease Research, Seattle, Washington, USA. ${ }^{3}$ Divisions of Nephrology and Pulmonology, Departments of Medicine and Pathology, University of Washington, Seattle, Washington, USA

\begin{abstract}
Fibrotic disease is associated with matrix deposition that results in the loss of organ function. Pericytes, the precursors of myofibroblasts, are a source of pathological matrix collagens and may be promising targets for treating fibrogenesis. Here, we have shown that pericytes activate a TLR2/4- and MyD88-dependent proinflammatory program in response to tissue injury. Similarly to classic immune cells, pericytes activate the NLRP3 inflammasome, leading to IL-1 $\beta$ and IL-18 secretion. Released IL-1ß signals through pericyte MyD88 to amplify this response. Unexpectedly, we found that MyD88 and its downstream effector kinase IRAK4 intrinsically control pericyte migration and conversion to myofibroblasts. Specific ablation of MyD88 in pericytes or pharmacological inhibition of MyD88 signaling by an IRAK4 inhibitor in vivo protected against kidney injury by profoundly attenuating tissue injury, activation, and differentiation of myofibroblasts. Our data show that in pericytes, MyD88 and IRAK4 are key regulators of 2 major injury responses: inflammatory and fibrogenic. Moreover, these findings suggest that disruption of this MyD88-dependent pathway in pericytes might be a potential therapeutic approach to inhibit fibrogenesis and promote regeneration.
\end{abstract}

\section{Introduction}

Fibrotic diseases can affect any organ or tissue in the body and represent a leading cause of morbidity and mortality (1). Despite this, therapies are relatively ineffective, in part because they do not directly target the mechanism of fibrosis. Therefore, better understanding of responses to injury specifically in matrix-depositing cells is important for identifying potential therapeutic targets directed at restoring organ function. Previously, there have been reports that myofibroblasts can be derived from multiple cell types, including epithelium and leukocytes (2-4). Recent studies have indicated that the kidney has a large population of resident stromal cells lining the peritubular capillaries, known as pericytes, and they represent a major source of interstitial myofibroblasts in renal fibrogenesis, which deposit pathological matrix $(1,5-8)$. Pericytes have been implicated in skin as highly sensitive to local tissue injury and as guardians of extravasation of leukocytes to the site of tissue injury $(9,10)$. Additionally, there have been multiple reports on the role of pericytes in the lung, retina, skeletal muscle, cancers, and Alzheimer's disease, presenting strong evidence that these cells might be promising targets for therapy (11-17).

Injurious signals activate a range of responses in multiple cell types. Damaged epithelium generates TGF- $\beta$ and other signals that promote wound healing (18). Injured or dying cells release products, known as alarmins or damage-associated molecular patterns (DAMPs), detected by immune receptors in immune

Conflict of interest: I.A. Leaf, B.G. Johnson, K.M. Guckian, I.G. Gomez, and J.S. Duffield own Biogen stock. K.M. Guckian, I.A. Leaf, and J.S. Duffield have filed a patent for the use of IRAK4 inhibition in the treatment of fibrosis (WO 2016011390 A1).

Submitted: March 15, 2016; Accepted: October 13, 2016.

Reference information: / Clin Invest. 2017;127(1):321-334. doi:10.1172/JCI87532. and nonimmune cells (19). Surveillance mechanisms involve pattern recognition receptors: on the cell surface Toll-like receptors (TLRs) and in the cytoplasm NOD-like receptors, triggering activation of the innate immune response that promotes the recruitment and activation of leukocytes, critical for eliminating foreign particles and host debris. Cell adhesion molecules and chemokines facilitate leukocyte extravasation from the circulation to the affected site and initiate signals that regulate leukocyte motility and effector functions. Myeloid differentiation primary response gene 88 (MyD88) is an adapter molecule important for transduction of many of these injury signals and induction of genes encoding enzymes, chemokines, cytokines, adhesion molecules, and regulators of the extracellular matrix. A subset of cytosolic pattern recognition receptors, known as NOD-like receptors (NLRs), activates the protease caspase-1, which causes rapid maturation/secretion of preformed cytokines including IL-1 and IL-18 as well as inducing proinflammatory cell death, pyroptosis (20, 21). Although this process is a natural response directed at wound healing and repair, in the case of impaired resolution, or persistent triggers in chronic disease, excessive inflammation and matrix deposition lead to pathology and organ dysfunction.

Here we investigate the mechanism of response to ischemic injury specifically in matrix-depositing stromal cells, pericytes, and, using in vitro and in vivo studies, demonstrate that pericyte MyD88 is a key regulator of injury responses. In pericytes, MyD88 controls immune signaling, which enables detection and amplification of the inflammatory signal; and simultaneously activates fibrogenesis, contributing to matrix deposition and pathology. We also show that pharmacological inhibition of MyD88 signaling by a novel IRAK4 inhibitor in vivo is protective in kidney injury and, therefore, could be a promising new therapy in postinjury care. 
A

Pathways enriched in AKI

Response to wounding

Inflammatory response

Defense response

Acute inflammatory response Immune response

Immune effector process Integrin-mediated signaling

$$
\begin{array}{llllllll}
0 & 2 & 4 & 6 & 8 & 10 & 12 & 14 \\
-\log P & & & &
\end{array}
$$

B
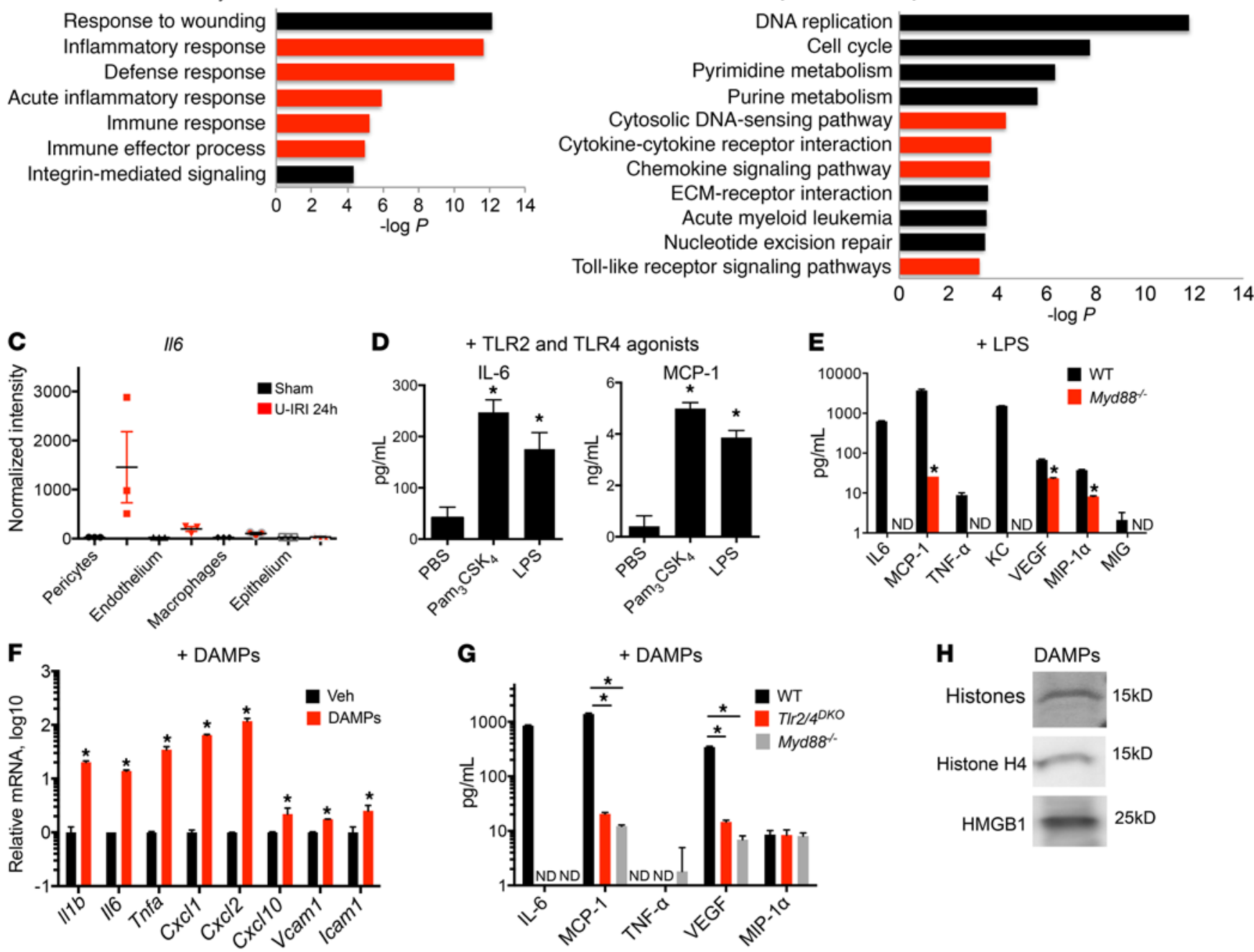

G

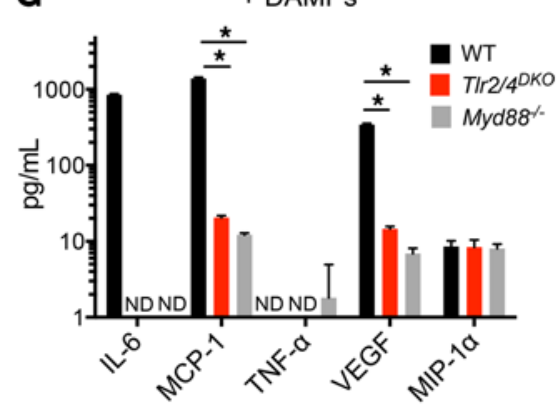

J
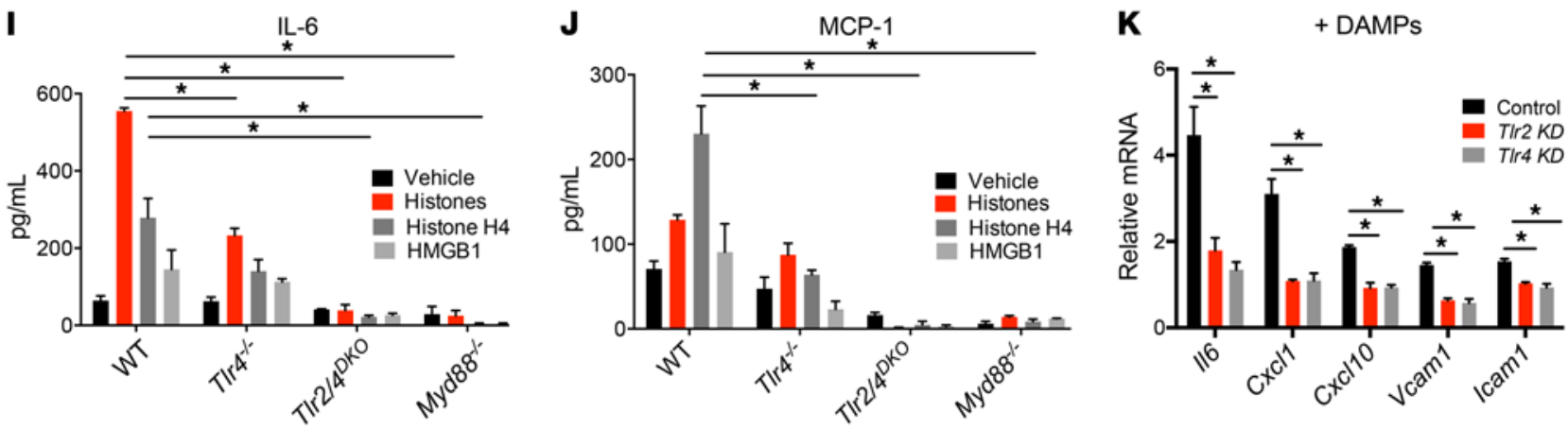

Figure 1. TLR2/4- and MyD88-dependent injury responses in pericytes. (A and B) Enrichment analysis of biological process ontology in human biopsies from acute kidney injury (AKI) patients compared with healthy controls (A) and myofibroblasts from control and kidneys with acute injury (B). Innate immune-related pathways are highlighted in red. The $x$ axis of the graph shows -log $P$ values calculated using $t$ test for the enrichment of a specific pathway. ECM, extracellular matrix. (C) RNA expression levels of $I / 6$ in pericytes, macrophages, and epithelial and endothelial cells. Translated RNA was isolated from unilateral IRI (U-IRI) and sham control kidneys 24 hours after injury. The $y$ axis shows normalized intensity for II6 transcript in microarrays; $n=3$ per group. (D) Cytokine concentration in supernatants from cultured pericytes 24 hours after stimulation with TLR ligands. (E) Secretion in WT or Myd88 ${ }^{-/-}$pericytes 24 hours after treatment with LPS. (F) Transcriptional response of pericytes to diseased kidney DAMPs at 6 hours. (G) Cytokine con-

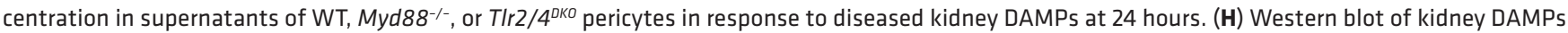

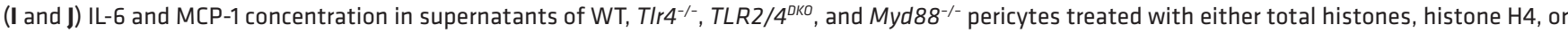
HMGB1 for 24 hours. (K) Effect of silencing of TIr4 or TIr2 on pericyte response to kidney DAMPs. ( $n=3-6$ per group; ${ }^{*} P<0.05,1$-way and 2 -way ANOVA, Bonferroni's multiple comparisons test.) 


\section{Results}

Pericytes respond to injury in a MyD88-and TLR-dependent manner. Following acute kidney injury, human kidney either regenerates, or develops fibrotic disease. We performed gene ontology analysis of the transcriptional profiles of biopsies from patients with acute kidney injury (22), using the Database for Annotation, Visualization, and Integrated Discovery (DAVID) $(23,24)$, and discovered strong enrichment for innate immune pathways among the top upregulated pathways associated with disease (Figure 1A and Supplemental Table 1; supplemental material available online with this article; doi:10.1172/JCI87532DS1). Cell-specific analysis of injured mouse kidneys with fibrosis using a method known as translating ribosome affinity purification (TRAP) revealed an overwhelming number of innate immunity-related pathways, including cytosolic DNA sensing, cytokine-cytokine interactions, chemokine signaling, and TLR and NLR signaling (Figure 1B and Supplemental Table 2), as being specifically upregulated during transition of pericytes to pathological myofibroblasts. This transition is associated with increased cellular proliferation and extracellular matrix deposition, eventually leading to metabolic disease and loss of kidney function. Consistently with these changes, among the most enriched pathways in myofibroblasts were DNA replication, cell cycle, extracellular matrix-receptor interaction, and pyrimidine and purine metabolism (Figure 1B). IL-6 is a major proinflammatory cytokine secreted during tissue damage. To our surprise, pericytes had significantly higher expression of Il6 compared with endothelial and epithelial cells and even macrophages in mouse kidneys following unilateral ischemiareperfusion injury (IRI), which is a good model for human acute kidney injury (Figure 1C) (25). Together these in vivo findings suggested that innate immune responses may be important determinants of the fibrotic response to tissue injury and pericytes are the cells that initiate response to tissue injury, and active innate immune signaling in these cells contributes to disease progression.

To characterize innate immune signaling in pericytes, primary mouse kidney pericytes were isolated as previously reported (8), and response to TLR ligands was detected by measurement of IL-6 and monocyte chemoattractant protein-1 (MCP-1) secretion. Pericytes express transcripts for TLRs and signaling adapters (Supplemental Figure 1, A and B). Interestingly, TLR1/2 and TLR4 as well as TLR2/6, TLR3, TLR7, and TLR9 were competent in sensing their corresponding ligands, though TLR5 was not (Figure 1D and Supplemental Figure 1C). Activation of TLR4 with LPS resulted in transcription and secretion of a broad array of inflammatory factors by pericytes, and this response was dependent on MyD88 (Figure 1E). Similar responses were recapitulated in primary human kidney pericytes (Supplemental Figure 1D).

To model responses to ischemic tissue injury in vitro, we used DAMPs released from injured kidneys by collecting the soluble fraction of extracellular molecules 24 hours after IRI; a time corresponding to the peak of innate immune response and necrosis associated with injury (Supplemental Figure 2) (26). Culturing pericytes with kidney DAMPs induced transcription of proinflammatory cytokines $I l 6, I l 1 b$, and Tnfa; chemokines Ccl2 (also known as Mcp-1), Cxcl1 (also known as KC), and Cxcl2 (also known as Mip2); and adhesion molecules Icam1 and Vcam1 (Figure $1 F)$. TLR2 and TLR4 have been previously shown to play import- ant roles in response to DAMPs and to require MyD88 for signal transduction $(27,28)$. Pericytes responded to kidney DAMPs by secretion of a variety of cytokines, chemokines, and growth factors, most of which were exclusively controlled by MyD88, TLR2, and TLR4 (Figure 1G). We show that these injury responses in pericytes are conserved between mouse and human, as human kidney pericytes showed similar responses to LPS and DAMPs (Supplemental Figure 1, D and F). DAMP activity was markedly diminished by proteases rather than nucleases (Supplemental Figure 2, A and B), suggesting proteins are the major components of the DAMPs that are recognized by pericytes. Mass spectrometric analysis of DAMPs indicated significant enrichment for histones, nuclear proteins previously reported to be endogenous DAMPs (Supplemental Figure 2). Some of the known DAMPs, such as high-mobility group protein B1 (HMGB1) as well as nuclear proteins including histones, particularly $\mathrm{H} 4$, have been shown to be released by injured cells and to activate TLR2 and TLR4 $(27,28)$. Protein lysates of disease DAMPs showed significant levels of total histones, H4, or HMGB1, similar to mass spectrometry (Figure $1 \mathrm{H}$; see complete unedited blots in the supplemental material). Incubation of pericytes with total histones, H4, or HMGB1 led to secretion of IL-6 and MCP-1, a partial response in $\mathrm{Tlr} 4^{-/-}$pericytes, and complete ablation of secretion in $\mathrm{Tlr} 2 / 4^{\mathrm{DKO}}$ and $\mathrm{Myd} 88^{-/-}$pericytes (Figure 1I). Similarly, silencing of Tlr2 or Tlr4 reduced responsiveness to DAMPs (Figure 1, J and K, and Supplemental Figure 1G). To validate further the role of pericytes as innate immune sentinels against tissue damage, comparative experiments in vitro suggested that pericytes were more sensitive to DAMPs than primary epithelial cells, or monocyte-derived macrophages (Supplemental Figure 1, H and I). We therefore conclude that pericytes are highly sensitive to tissue injury and respond to endogenous DAMPs released from injured cells through activating TLRs and MyD88.

Kidney DAMPs activate the NLRP3 inflammasome in pericytes, leading to $I L-1 \beta$ secretion. The major proinflammatory cytokines IL-1 $\beta$ and IL-18 require a second signal, generated by a cytosolic NLR, to be cleaved to their mature forms and secreted from the cell (21). Activated NLRs will oligomerize and recruit pro-caspase-1 to form an inflammasome that will process pro-IL-1 $\beta$ and pro-IL-18 for secretion. We show here that the NLR signaling pathway is enriched in myofibroblasts during response to injury in vivo (Supplemental Table 2). In vitro, pericytes secrete IL-1 $\beta$ and IL-18 when primed with LPS and stimulated with extracellular ATP, and this secretion is completely abolished in $\mathrm{Nlrp} 3^{-/-}$and Casp1/11 ${ }^{\text {DKO }}$ pericytes, implicating activation of the NLRP3 inflammasome (Figure 2A). Pericytes primed with LPS accumulate pro-IL-1 intracellularly and release cleaved mature IL-1 $\beta$ with addition of ATP (Figure 2B). This activity is associated with increased NLRP3 expression, perhaps due to increased stability of the protein once it is assembled in an inflammasome complex (Figure 2C). NLRP3 inflammasome activation in response to sterile injury has been implicated in several noninfectious disease models (29), and next we tested whether pericytes activate the NLRP3 inflammasome in response to kidney DAMPs. As predicted, IL-1 $\beta$ and IL-18 were secreted by pericytes cultured with DAMPs, and this secretion was dependent on the presence of NLRP3 and caspase-1/11 (Figure 2, D and E). The shortlived tissue injury molecule and inflammasome activator, ATP markedly enhances this DAMP response (Figure 2, C-E). Moreover, 
A

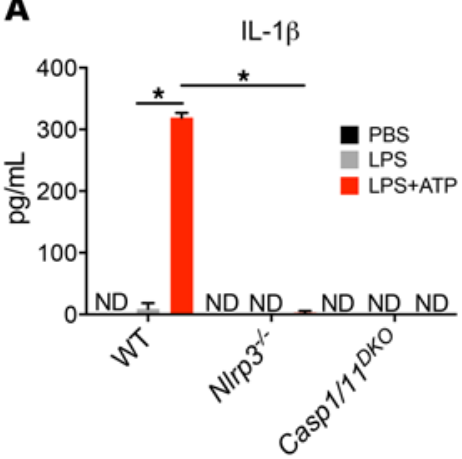

B

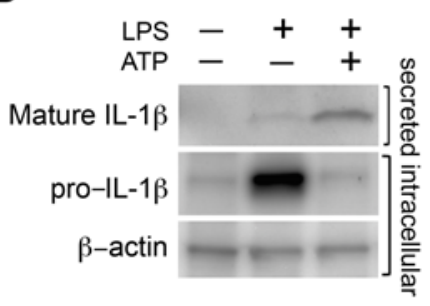

C

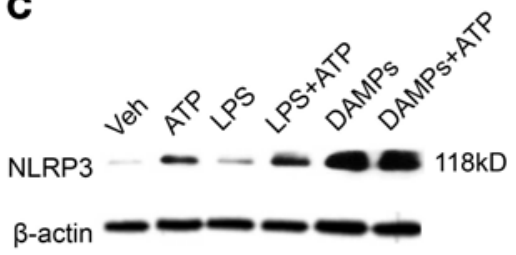

D

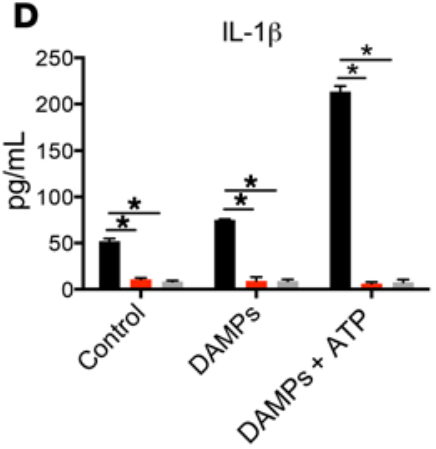

E

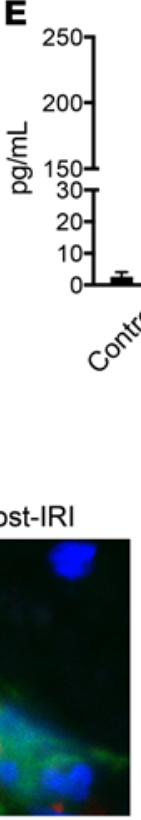

Day 1 post-IRI
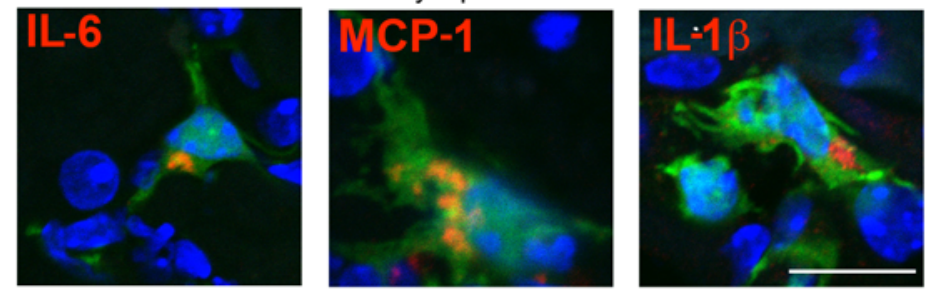

PERICYTES NUCLEI
H $+\mathrm{IL}-1 \beta$
I

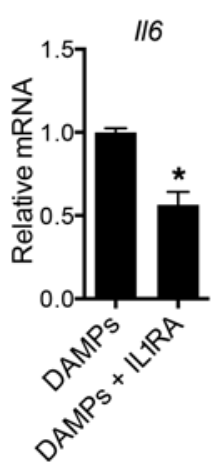

J

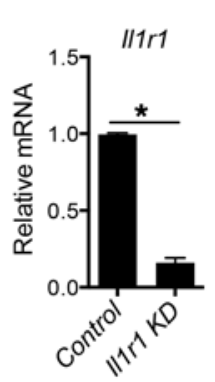

F + DAMPs

IL-1 $\beta$

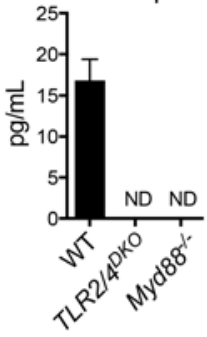

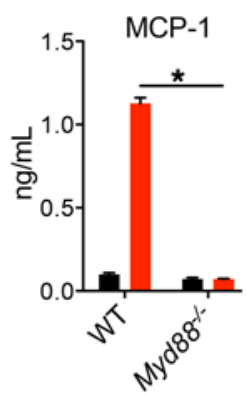

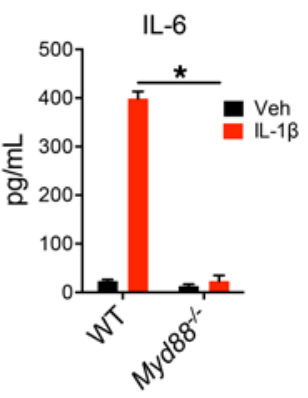

K

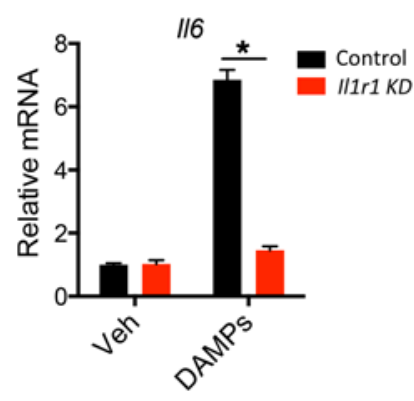

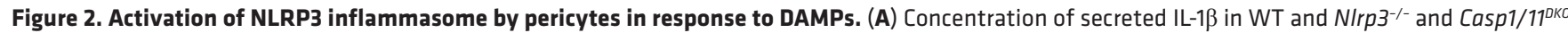
pericytes 24 hours after treatment with LPS and ATP. (B) Western blot of intracellular pro-IL-1 $\beta$ and secreted mature IL-1 $\beta$ from pericytes 24 hours after treatment with LPS and ATP. (C) Western blot of pericyte cell lysates 24 hours after treatment with LPS, ATP, and DAMPs. (D and E) IL-1 $\beta$ and IL-18 concentration in WT and mutant pericyte supernatants after treatment with DAMPs and ATP. (F) IL-1 $\beta$ concentration in supernatants of WT, TLR2/4סKo,

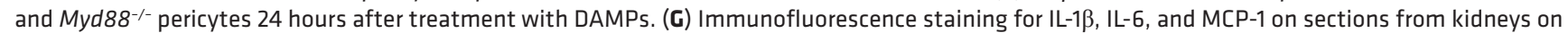
day 1 after IRI. (H) IL-6 and MCP-1 cytokine response to IL-1 $\beta$ treatment in the supernatant of WT and Myd88-- pericytes. (I) The effect of recombinant IL-1 receptor antagonist (IL1RA) on transcriptional response to DAMPs. (J and K) The effect of siRNA against I/1r1 on I/1r1 expression (J) and I/6 response to DAMPs (K). ND, not detected. KD, knockdown. (Scale bar: $25 \mu \mathrm{m} ; n=3-6$ per group; ${ }^{*} P<0.05$, 2-tailed Student's $t$ test or 2 -way ANOVA, Bonferroni's multiple comparisons test.) 
IL-1 $\beta$ production in response to DAMPs required MyD88- and TLR2/4-dependent priming (Figure 2F). We further confirmed the relevance of this innate immune activation in vivo by staining kidney sections of control (sham-operated) and experimental mice 1 day after IRI with antibodies for proinflammatory cytokines and chemokines. While control kidneys had no detection of these immune activation markers, injured kidneys showed intracellular accumulation of IL-6, IL-1 $\beta$, and MCP- 1 in pericytes (Figure $2 \mathrm{G}$ ).

Pericytes respond to $I L-1 \beta$ signaling by amplifying inflammation. Recently, it was reported that IL-1 receptor activation can lead to severe renal epithelial cell damage in vitro and exacerbation of fibrotic renal disease due to increased proinflammatory cytokine release; however, other studies had reported a minor role for IL-1 receptor $(30,31)$. Similarly to the TLR response, IL-1 signaling also requires MyD88 for signal transduction (32). In addition to IL-1 $\beta$, other cytokines from the IL-1 superfamily, such as IL-18 and IL-33, activate IL-1 receptor and require MyD88. Pericytes upregulate expression of the IL-1 receptor Il1r1 in vivo following ischemic injury (Supplemental Figure 3, A and B). To test whether pericytes respond to IL-1 cytokines, we treated them with exogenous, recombinant IL-1 $\beta$ and detected a strong response by measuring secretion of IL- 6 and MCP-1, demonstrating that pericytes have active IL-1 signaling, and this response is dependent on MyD88 (Figure $2 \mathrm{H}$ and Supplemental Figure 3C). Therefore, DAMP-treated pericytes not only release IL-1 $\beta$ but are also able to respond to this cytokine, thereby amplifying the inflammatory response. To characterize this autocrine response to IL-1 cytokines produced in response to injury, we activated pericytes with kidney DAMPs in the presence of IL-1 receptor antagonist (IL1RA) and showed a $43.5 \%$ reduction of Il6 expression (Figure 2I), emphasizing a role for IL-1 signaling in amplifying proinflammatory responses to injury. Silencing of Illr 1 in pericytes inhibited IL- 6 and MCP-1 reduction further, suggesting there may be a broader role for the receptor in pericyte activation than binding to IL-1 (Figure 2, J and K).

MyD88 regulates migratory and fibrotic responses in pericytes. Pericytes have been shown to play important roles in injury responses, by proliferating, migrating away from capillary walls, and activating an extracellular matrix deposition program $(6,33)$. Morphogens and developmentally regulated cytokines including PDGFs, WNTs, and the TGF- $\beta$ superfamily are well recognized to contribute to this process $(1,18)$. We tested whether MyD88 played a role in these responses. In a scratch-wound assay, mouse pericytes migrate in response to TGF- $\beta$ and upregulate the transcription of genes encoding the fibrotic matrix protein collagen I (Col1a1) and the contractile protein $\alpha$ SMA (Acta2), thereby mediating fibrot-

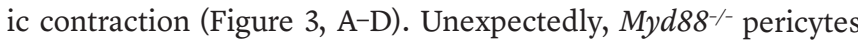
did not migrate in response to TGF- $\beta$ and expressed significantly less Col1a1 and Acta2 (Figure 3, A-D), thereby demonstrating that MyD88 is critical in TGF- $\beta$-induced pericyte activation. Similarly to TGF- $\beta$, kidney DAMPs induced pericyte differentiation into fibroblasts associated with increased migration, transcription of collagen I, and $\alpha$ SMA expression, and MyD88 was required for this differentiation (Figure 3, E-G). Interestingly, both responses to TGF- $\beta$ and kidney DAMPs were mediated by TLR2 and TLR4 as demonstrated by the failure of $T l r 2 / 4^{D K O}$ pericytes to migrate or express fibroblast markers (Figure $3, \mathrm{~A}-\mathrm{G}$ ), or the observation that silencing of Tlr2 or Tlr4 reduced pericyte expression of collagen I
(Figure 3H). These findings directly implicate MyD88, TLR2, and TLR4 in the fibrogenic process.

We sought to test whether this mechanism is evolutionarily conserved. Human pericytes treated with TGF- $\beta$, kidney DAMPs, or histones became activated and formed stress fibers visualized by staining with phalloidin, migrated to close a scratch wound, and induced expression of COL1A1 and ACTA2 (Figure 3, I-K, and Supplemental Figure 4A). Pharmacological inhibition of MyD88 using an inhibitor peptide that blocks homodimerization of MyD88 reduced myofibroblast differentiation (Figure 3, J and $\mathrm{K})$ as well as expression of fibroblast markers in response to both TGF- $\beta$ and DAMPs (Supplemental Figure 4, B and C).

To understand the mechanism of the MyD88/TLR2/TLR4 regulation of fibrotic responses, we first tested whether previously reported antagonism between NF- $\kappa$ B and TGF- $\beta$ pathways via the decoy receptor BAMBI or the inhibitory adapter SMAD7 was operative $(34,35)$. Neither expression of BAMBI nor of SMAD7 was increased in the absence of TLR2/4 (Supplemental Figure 5, A and $\mathrm{B})$, demonstrating that inhibition of TGF- $\beta$ and DAMP responses by TLR2/4 and MyD88 is mediated via a novel mechanism.

To dissect this novel mechanism, we tested whether MyD88-mediated responses require TGF- $\beta$ signaling. While a pan-neutralizing antibody against TGF- $\beta$ inhibited TGF- $\beta$ induced migration, it did not affect basal or DAMP-induced migration (Figure $3 \mathrm{~L}$ ), suggesting that in the latter case, TGF- $\beta$ is not required for pericyte activation and migration is either ligand-independent or directly induced by other ligands such as histones, etc (36). On the other hand, blocking TGF- $\beta$ receptor I and II (TGFßRI/II) activation using the dual kinase inhibitor LY2109761 significantly inhibited basal, TGF- $\beta$-induced, or DAMP-induced migration as well as expression of smooth muscle actin and collagen I in human pericytes (Figure 3, L and M, and Supplemental Figure 5C). Consistent with this crosstalk between MyD88 and TGF $\beta$ RI/II pathways, protein levels of MyD88 are rapidly increased following TGF- $\beta$ treatment. Silencing of MyD88 notably increased levels of TGF $\beta$ RI and the $46-\mathrm{kDa}$ isoform of JNK at baseline that were reduced in response to TGF- $\beta$ while not affecting their active phosphorylated forms (Supplemental Figure $5 \mathrm{D})$. Therefore, convergence of NF- $\mathrm{KB}$ and TGF- $\beta$ signaling occurs upstream at the receptor level, and we show that TGF- $\beta$ receptor, TLR2/4, and MyD88 are required for fibrotic responses to injury.

Neither stimulation by IL-1 $\beta$ nor inhibition by IL1RA of pericytes had any effect on fibroblast differentiation (Supplemental Figure 3, D-J), but silencing of Il1r1 reduced DAMP-stimulated collagen I expression (Supplemental Figure 3, F and G). One possible explanation for this discrepancy between receptor blockade and receptor silencing is that IL1R1 may contribute in a ligandindependent manner to a profibrotic signaling complex. Nevertheless, IL-1 signaling predominantly affects inflammatory signaling by pericytes, and not profibrotic differentiation (36).

Deletion of Myd88 in stromal cells is protective against ischemic injury in vivo. We previously reported that deletion of $M y d 88$ has a drastic effect and significantly alleviates inflammation and fibrogenesis $(26,37)$. Although macrophages have been extensively studied as classic immune cells recognizing danger signals and responding by turning on inflammatory responses, deletion of $M y d 88$ specifically in myeloid lineage did not affect 

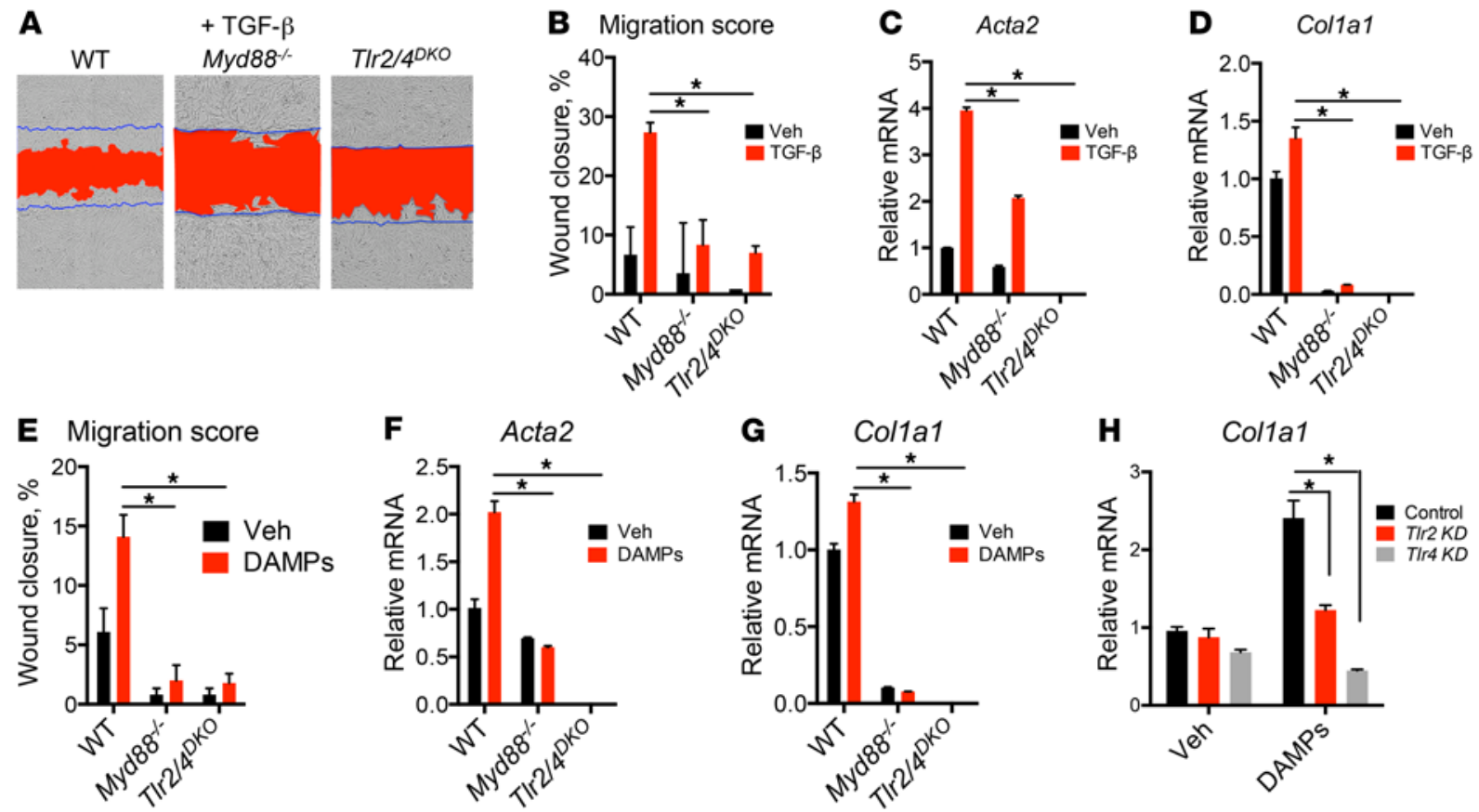

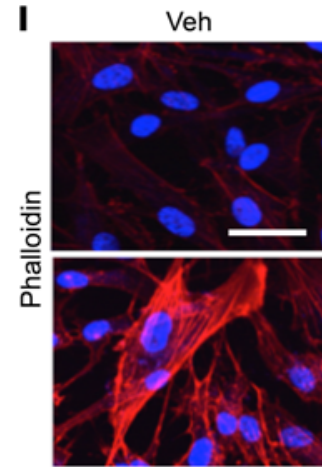

Histones

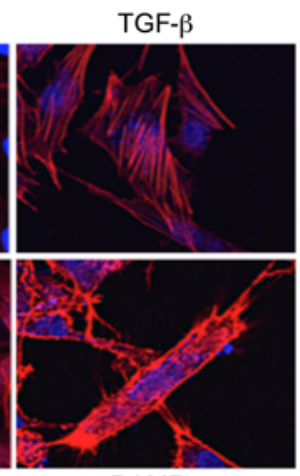

DAMPs
J

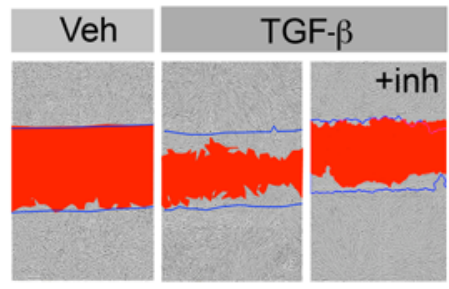

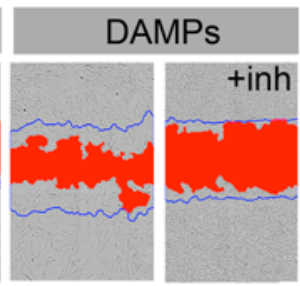

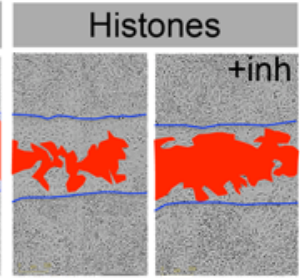

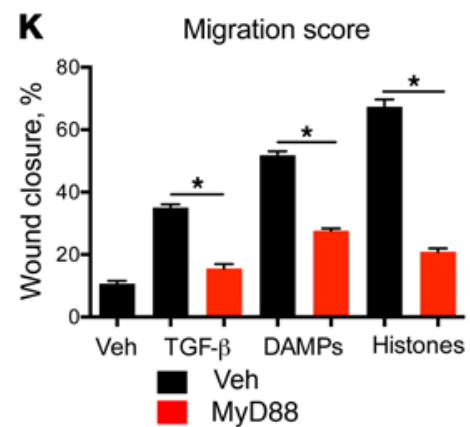
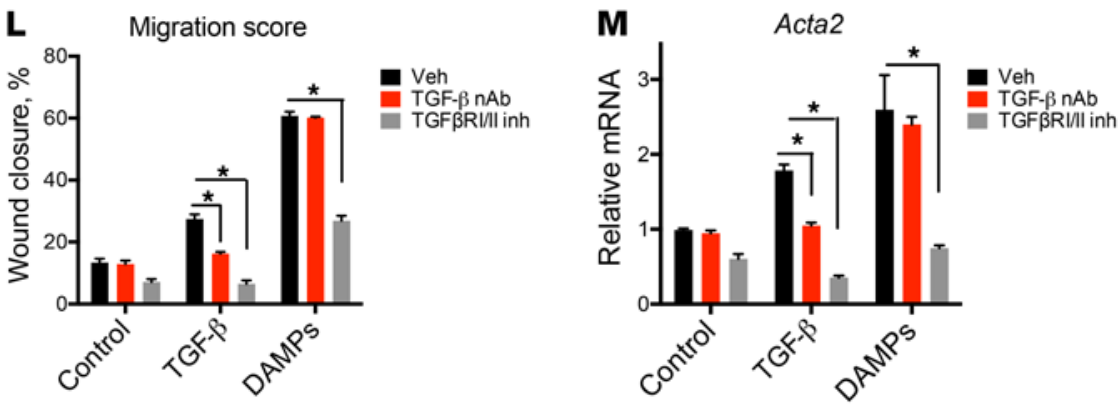

Figure 3. TLR2/4 and MyD88 control fibrogenic responses of mouse and human pericytes. (A) Representative images showing migratory response of WT, Myd88 $8^{-/-}$, and Tlr2/4 ${ }^{D K O}$ pericytes to 24-hour TCF- $\beta$ treatment. Blue lines mark the boundaries of the scratch at 0 hours; red area designates scratch boundaries at 24 hours. (B) Quantification of TGF- $\beta$ migration assays. (C and D) Transcription of Acta2 and Col1a1 induced by TGF- $\beta$. (E-G) Responses of WT, Myd88 $8^{-/-}$, and TIr2/4 ${ }^{\text {KKO }}$ pericytes at 24 hours to kidney DAMP treatment. (E) Quantification of migration. (F and G) Transcription of Acta2 and Col1a1. (H) Effect of Tlr2 or Tlr4 silencing with siRNA on upregulation of Col1a1 stimulated by DAMPs. (I) Stress fiber formation shown by phalloidin staining in human kidney pericytes treated with TCF- $\beta$, histones, or kidney DAMPs. (J and K) Representative images (J) and quantification (K) of migratory response of human pericytes to TCF- $\beta$, histones, or DAMPs in the presence of a MyD88 inhibitor or vehicle. (L) Quantification of migration induced by TCF- $\beta$ or DAMPs in the presence or absence of TGF- $\beta 1,2,3$ neutralizing antibody (NAb) or TCFR $\beta$ I/II inhibitor LY2109761, 24 hours after treatment. (M) Transcription of myofibroblast marker Acta2 in response to TGF- $\beta$ or DAMPs in the presence or absence of the TGFR 3 I/II inhibitor LY2109761, in mouse pericytes after 24-hour treatment. (Scale bar: $25 \mu \mathrm{m} ; n=3-6$ per group; ${ }^{*} P<0.05$, 2-tailed Student's $t$ test or 2 -way ANOVA, Bonferroni's multiple comparisons test.) 

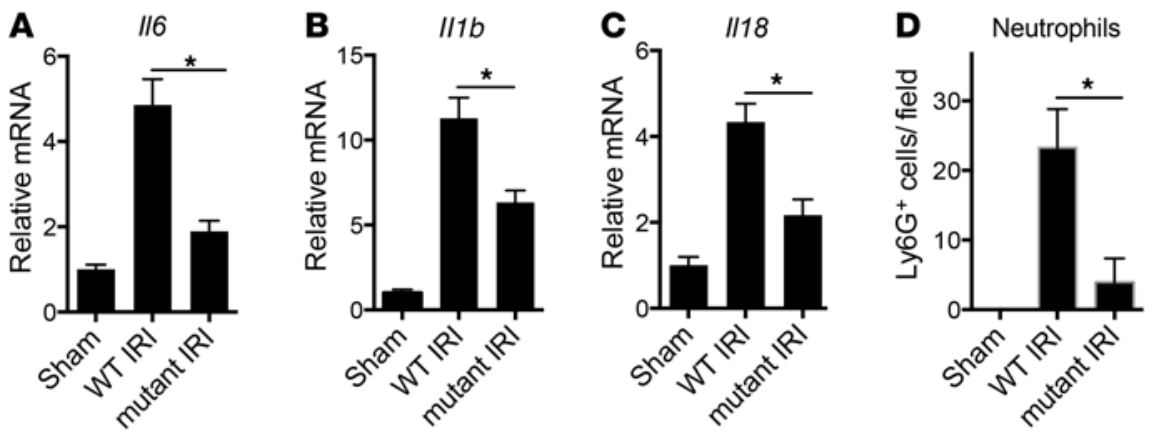

Figure 4. Myd88 deletion specifically in pericytes attenuates injury and fibrotic responses following IRI. (A-C) Transcription of inflammatory genes in kidneys from control and day 5 post-IRI kidneys from WT and Foxd1 ${ }^{+/ \text {/re }}$ Myd8 $88^{f l / f l}$ (referred to as mutant) mice. (D) Quantification of Ly6G+ neutrophils on kidney sections. (E-H) Representative images of kidney sections stained with Ly6C antibody, TUNEL, PAS, or Sirius red. (F) TUNEL ${ }^{+}$ cells are labeled green. (C) Necrotic tubules in PAS-stained sections are labeled with arrowheads. (H) Fibrosis is detected as red stain. (I-K) Graphs quantifying apoptotic (TUNEL+) cells (I), tubular injury score based on PAS-stained sections (J), and fibrosis score based on Sirius red staining (K). ( $\mathbf{L}$ and $\mathbf{M}$ ) Transcription of Col1a1 and Acta2. (Scale bars: $50 \mu \mathrm{m} ; n=6$ per group; ${ }^{*} P<0.05$, 2-tailed Student's $t$ test or 2-way ANOVA, Bonferroni's multiple comparisons test.)

E

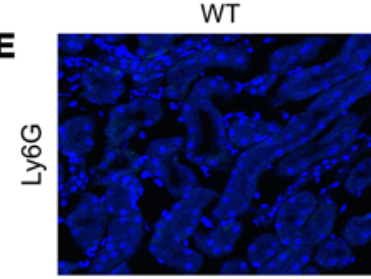

$\mathbf{F}$

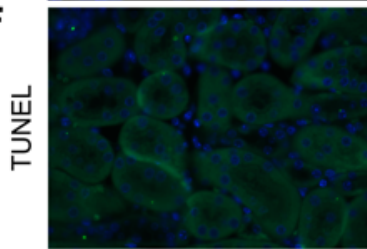

G

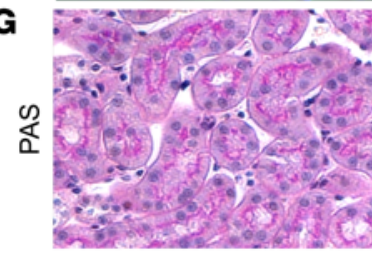

H

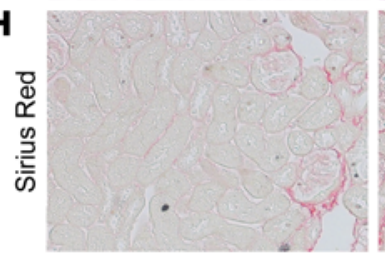

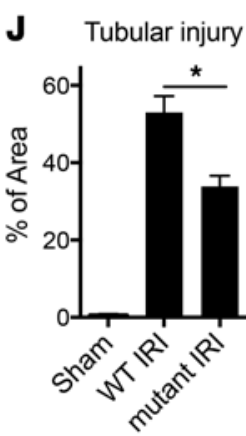

K Fibrosis

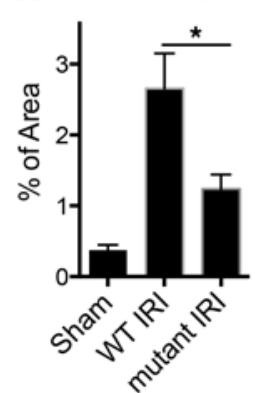

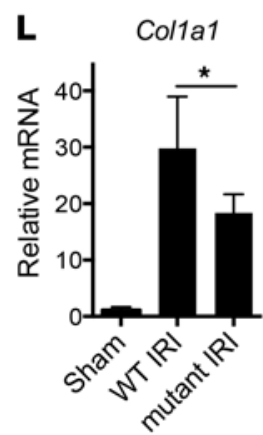

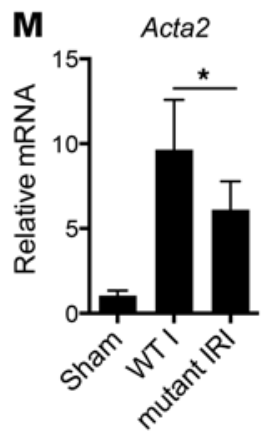

ischemic injury severity or progression of wound healing (ref. 26 and Supplemental Figure 6). The results presented here suggested that in fibrotic disease MyD88 may be functioning dominantly in the stromal lineage. We therefore deleted Myd88 specifically in pericyte precursors using Foxd1 $1^{+/ C r e}$ Myd8 $88^{f l o x / f l o x}$ mice $(6,38,39)$. This lineage has been confirmed not to con- tain myeloid or epithelial cells (6). Loss of MyD88 protein in adult kidney in myofibroblasts was confirmed by PCR showing recombination between loxP sites resulting in excision of exon 3 of Myd88. Adult kidneys from mutant mice appear normal (Supplemental Figure 6, A-C). Mice were subjected to kidney IRI and evaluated 5 days after surgery for injury, fibrotic, and 

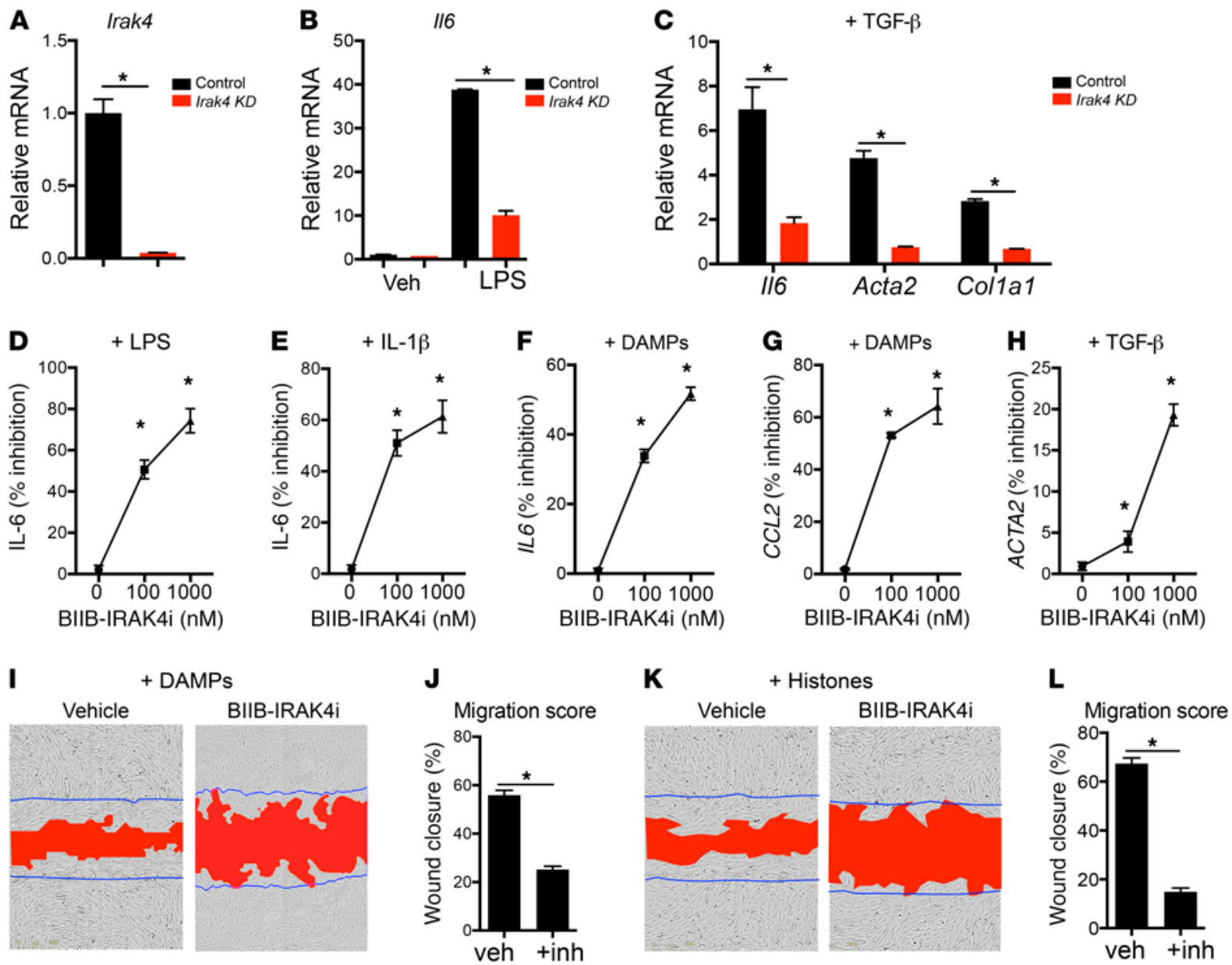

Figure 5. Role of IRAK4 in pericytes and its inhibition in vitro by BIIB-IRAK4i. (A) Irak4 transcript levels in pericytes after treatment with either scramble or Irak4-specific siRNA. (B) II6 transcript levels in control and Irak4 knockdown (KD) pericytes 24 hours after treatment with LPS. (C) II6, Col1a1, and Acta2 expression in control and Irak4 KD pericytes 24 hours after TCF- $\beta$ treatment. (D-H) Inhibition of injury responses by IRAK4 inhibitor BIIB-IRAK4i in kidney pericytes in vitro. (D and E) Inhibition of IL-6 secretion by mouse kidney pericytes stimulated with LPS (D) or IL-1B (E) in the presence of BIIB-IRAK4i. (F and G) Inhibition of IL6 and CCL2 transcription by human kidney pericytes in response to kidney DAMPs. (H) Inhibition of ACTA2 transcription by human kidney pericytes following TGF- $\beta$ treatment. (I-L) Inhibition of pericyte migration by IRAK4 inhibitors. (I and K) Representative images of human pericytes stimulated with kidney DAMPs (I) or histones (K) and either vehicle or IRAK4 inhibitors in scratch-wound assays. Blue lines mark the scratch boundaries at 0 hours, and red area designates the scratch at 16 hours. ( $\mathbf{J}$ and $\mathbf{L})$ Migration scores of DAMP-induced (J) or histone-induced $(\mathbf{L})$ migration. $\left(n=3-6\right.$ per group; ${ }^{*} P<0.05,2$-tailed Student's $t$ test, 1-way or 2-way ANOVA, Bonferroni's multiple comparisons test.)

inflammatory responses (Figure 4). WT mice had severe disease as indicated by increased inflammatory markers, neutrophil influx (Figure 4, A-E), increased apoptosis (Figure 4, F and I), epithelial injury with numerous necrotic tubules (Figure 4, $\mathrm{G}$ and $\mathrm{J}$ ), high fibrotic score and collagen I expression (Figure $4, \mathrm{H}, \mathrm{K}$, and $\mathrm{L}$ ), and activation and expansion of myofibroblasts (Figure 4M and Supplemental Figure 4D).

Consistent with the role of MyD88 in innate immune signaling, deletion of Myd88 in pericytes only, significantly reduced inflammation as demonstrated by significant reduction in transcription of cytokines including $I l 6, I l 1 b$, and $I l 18$ (Figure 4, A-C) and reduced neutrophil recruitment (Figure 4, D and E). MyD88 has been shown

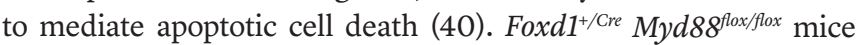
demonstrated improved tissue integrity and reduced cell death (Figure 4, F, G, I, and J). Further confirming our findings of a MyD88 role in myofibroblast differentiation, these $M y d 88$ mutant mice also showed reduction in fibrosis measured by Sirius red staining and reduced Col1a1 transcription, as well as expansion and differentiation of myofibroblasts as assessed by $\alpha$ SMA gene and protein expression (Figure 4, H and K-M, and Supplemental Figure 4D). Because of the potential role of epithelial or myeloid cells in innate immune sensing following initial kidney damage, we deleted Myd88 specif-

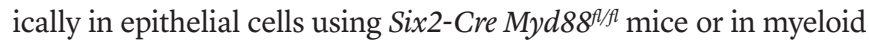

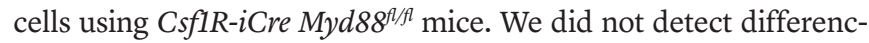
es in disease extent or inflammatory responses in comparison with WT mice (Supplemental Figure 6, E and F).

Inhibition of IRAK4 is protective against ischemic injury in vivo. We observed that multiple signaling pathways converge on MyD88 to promote inflammation and fibrosis in pericytes. We reasoned that signaling adapters downstream of MyD88 might be good pharmacological targets. In macrophages, IRAK4 is a serine/threonine protein kinase that is rapidly recruited by MyD88 following activation of TLR or IL-1 receptor and plays a critical role in innate immune response and activation of NF- $\mathrm{KB}$ 

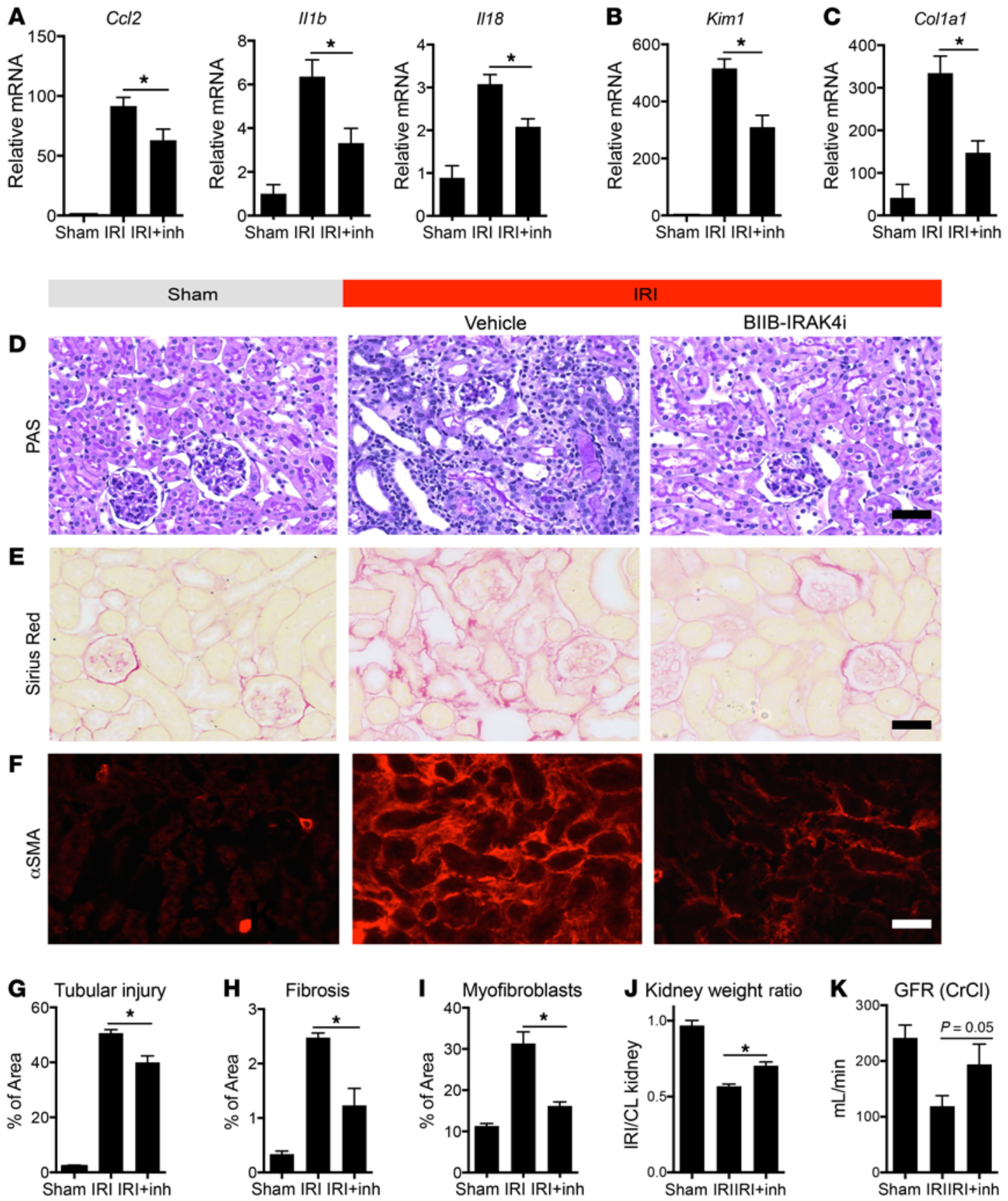

Figure 6. IRAK4 inhibition attenuates inflammatory and fibrotic responses in vivo and improves organ function. (A-C) Effect of kidney IRI and BIIB-IRAK4i (inh) on whole-kidney transcriptional responses at 7 days after injury showing inflammatory genes (A), epithelial injury marker (B), and Col1a1 (C). (D-I) Representative histological and immunofluorescence images (D-F) and quantitative analysis of kidneys from IRI at day 7 after injury treated with BIIB-IRAK4i compared with vehicle or sham control kidneys. (G-I) Quantification of tubular injury, interstitial fibrosis, and extent of myofibroblasts detected by aSMA. (I) Quantification of kidney weight changes associated with unilateral IRI. Ratios of ischemic kidneys' to contralateral (CL) kidneys' weights 14 days after injury are shown. (K) Measurement of glomerular filtration rate (GFR) by creatinine clearance (CrCl) in post-IRI kidney at 16 days, following contralateral nephrectomy at 14 days. (Scale bars: $50 \mu \mathrm{m} ; n=3-11$ per group; ${ }^{*} P<0.05$, 2-tailed Student's $t$ test or 2-way ANOVA, Bonferroni's multiple comparisons test.)

signaling (41). We first confirmed that pericytes express Irak4, then silenced Irak4 in mouse pericytes and showed that basal, LPS-induced, and TGF- $\beta$-induced inflammatory and profibrotic responses require Irak4 (Figure $5, \mathrm{~A}-\mathrm{C}$, and Supplemental Figure 7, A and B). To investigate whether inflammatory and fibrotic responses to injury in human pericytes can be pharma- cologically inhibited by targeting of MyD88-dependent signaling, we generated a novel small-molecule inhibitor of IRAK4 with exceptionally high potency and specificity. The novel small molecule, designated BIIB-IRAK4i, potently blocks phosphorylation of IRAK4 in vitro with an $\mathrm{IC}_{50}$ of $1 \mathrm{nM}$ (Supplemental Figure 8, A and B). BIIB-IRAK4i showed a very high affinity 


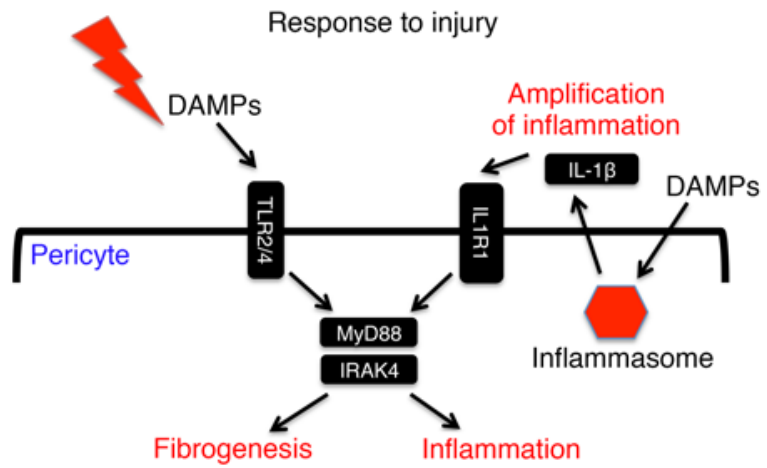

Figure 7. Schematic of injury sensing by MyD88 in pericytes. Pericytes sense tissue injury through TLR2 and TLR4. Both MyD88 and IRAK4 are important in transducing this signal and activation of inflammatory responses. Injurious molecules also activate inflammasome and release of additional proinflammatory cytokines including IL-1 $\beta$. Pericytes respond to $\mathrm{IL}-1 \beta$, and this generates an autocrine loop that amplifies the inflammatory response. In addition to inflammatory responses, activation of the TLR/ MyD88/IRAK4 axis by tissue injury induces myofibroblast differentiation.

for IRAK4 with a $K_{\mathrm{D}}$ of $0.034 \mathrm{nM}$, and a screen of its activity in the DiscoverX 456-member kinase panel indicated it to be highly selective and most potently inhibit its target IRAK4, with a second ranked inhibition mapped to IRAK1, which is downstream of IRAK4 in the MyD88 signaling pathway (Supplemental Figure 8, A and B). In comparative studies, BIIB-IRAK4i had 230-fold higher affinity and 120-fold greater potency for IRAK4 over IRAK1.

In mouse primary kidney pericytes, BIIB-IRAK4i demonstrated significant inhibition of both TLR4 and IL1R1 responses, by $80 \%$ and $57 \%$, respectively (Figure $5, \mathrm{D}$ and E). IRAK4-mediated responses are conserved between mouse and human, as in human pericytes, IRAK4 inhibition significantly reduced MyD88mediated inflammatory responses to kidney DAMPs measured by reduction in IL6 and CCL 2 expression (Figure 5, F and G), TGF- $\beta$ induced pericyte activation as indicated by ACTA2 transcription (Figure 5H), and migratory responses (Figure 5, I-L). BIIB-IRAK4i had excellent pharmacokinetic properties in vivo with once-daily oral dosing (Supplemental Figure 7, C-E, and Supplemental Figure 8, C and D). At day 7 after IRI, BIIB-IRAK4i significantly protected against injury and fibrosis by reducing levels of proinflammatory chemokines and cytokines (Figure 6A), tubular injury (Figure 6, $\mathrm{B}, \mathrm{D}$, and $\mathrm{G}$ ), production and deposition of fibrotic matrix (Figure 6, C, E, and H), and pathological differentiation of myofibroblasts (Figure 6, F and I). In additional studies, the function of the kidney 16 days after injury was evaluated, by performance of nephrectomy of the healthy kidney 14 days after ischemic injury. BIIB-IRA$\mathrm{K} 4 \mathrm{i}$ resulted in preservation of kidney weight, and preservation of glomerular filtration rate assessed by creatinine clearance (Figure 6, J and K, and Supplemental Figure 8, J and K). Furthermore, after 14 days of treatment, BIIB-IRAK4i persistently suppressed inflammatory and fibrotic markers in kidney tissue (Supplemental Figure $9, \mathrm{~A}-\mathrm{C})$. To distinguish the role of IRAK4 inhibition in limiting initial injury severity versus chronic disease extent following the phase of regeneration, we delayed administration of the inhibitor for 24 hours after initial IRI. In these therapeutic studies, BIIB-IRA-
K4i similarly inhibited inflammatory markers as well as markers of epithelial injury and fibrosis (Supplemental Figure 9, D-F).

\section{Discussion}

Here we report a key role for MyD88 and IRAK4 signaling in nonimmune cells, pericytes, in driving fibrotic disease. MyD88 has been shown to control important responses in immune cells in TLR and IL-1 signaling, and here we demonstrate that it plays a similar role in these 2 important pathways in pericytes. While TLR2 and TLR4 were identified as important pericyte receptors recognizing DAMPs, MyD88 appears to form a central node processing signals from TLRs and amplifying inflammatory signals from IL-1 receptor, and we show here that in pericytes this signaling contributes not only to chemokine production, cytokine production, and leukocyte recruitment but also to fibrogenesis (Figure 7).

Controversy persists as to the origin of myofibroblasts. Work from Kalluri's and other laboratories points to myofibroblast appearance from epithelial cells or myeloid progenitors in addition to resident fibroblasts (2-4). While epithelial and myeloid lineages are capable of fibrogenic transdifferentiation, other laboratories, including Humphreys's, McMahon's, and our own (6, 7, 24), demonstrated using genetic fate-mapping studies that pericytes are fibroblast progenitors and make a major contribution to scar-forming myofibroblasts. The present studies focus on the mechanism and pathology of injury sensing by pericytes rather than the origin of myofibroblasts.

Until recently, leukocytes have been thought of as principal regulators of inflammatory response, but the findings presented here point to a nonimmune cell lineage that makes an important contribution in response to tissue injury. While deficiency in MyD88 showed a significant improvement in the IRI models of kidney injury, deletion of $M y d 88$ specifically in the myeloid lineage had no impact (26). This finding combined with our in vitro and in silico analysis of pericyte responses to injury (Figure 1) led us to wonder whether pericytes may be playing a key role in this injury model. Our hypothesis was confirmed when depletion of MyD88 in pericytes phenocopied the total deficiency of MyD88. In addition to playing a vital role in vascular homeostasis and angiogenesis $(42,43)$, pericytes have recently been recognized as important precursor cells for cells that deposit pathological matrix, and our studies highlight how activation of TLR2, TLR4, and MyD88 in pericytes contributes to this fibrogenic process. Although growth factors, morphogens, and hypoxia are well described as contributing to fibrosis by driving fibroblast activation, the observations in these studies suggest that simultaneous signaling through MyD88 and its adapter IRAK4 is a prerequisite for the pathological fibrogenic response. This observation may explain why abundance of growth factors and activation of similar growth factor signalling pathways in development and organogenesis do not result in fibrosis. Current models of fibrogenesis point to leukocytes initiating inflammatory response and signaling to fibroblasts, but our new observations lead to the hypothesis that inflammatory signaling and growth factor signaling converge within a single cell type to result in fibrosis, and MyD88 and IRAK4 play central roles in this process (Figure 7). Here we show that in the myofibroblast precursors both the profibrotic TGF- $\beta$ receptors and TLR/MyD88 are required for myofibroblast differentiation, and we suggest that 
these 2 major pathways may cooperate, whereby TLR and MyD88 transduce profibrotic signaling by TGF- $\beta$ receptor transactivation. One potential explanation for the dependence of TGF $\beta$ RI/II signaling on TLR2/4 and MyD88 is that this latter signaling pathway mediates ligand-independent activation of the TGF- $\beta$ receptor, as has been reported previously (36). Although further work is required to understand the molecular mechanism of MyD88 and TGF $\beta$ RI/II transactivation, this signaling event may offer the possibility of novel approaches to block pathological signaling.

While macrophages have a higher sensitivity and magnitude of response to pathogenic TLR agonists, we demonstrate that pericytes possess most immune receptors and pathways and are competent in a variety of immune responses with, perhaps, a reduced strength of such a response to pathogen ligands. The weaker response might be due to the nature of the tissue injury. While in the setting of pathogen exposure, the efficient response will be faster and stronger, in the setting of sterile tissue injury the magnitude of an inflammatory response has to be carefully controlled to prevent excessive tissue damage and simultaneously promote healing and regeneration. Pericytes seem to be specialized cells for sensing the tissue injury response combining injury sensing and wound healing properties (Figure 7). Since many cells appear to have innate immune sensing capacity, it is likely that in chronic disease settings many cell types may become activated through TLR and MyD88 signaling. Our studies, however, point to pericytes of mesenchymal lineage as the cells that most strongly sense and respond to initial tissue injury. Our experiments predict that pericytes in other organs may serve a sentinel role in response to tissue injury. Hepatic pericytes have been reported to be activated by TLR4 in the setting of soluble pathogenic ligands transported to the liver via the splanchnic circulation (44).

The findings presented here add to our understanding of TLR signaling in tissue injury and directly implicate MyD88-directed $\mathrm{NF}-\kappa \mathrm{B}$ signaling in the stromal compartment in the fibrogenic process, emphasizing a critical role for pericytes, cells of mesenchymal origin, in the innate immune response to tissue injury, and may explain the reported association of TLR, IL-1, and IRAK1 polymorphisms with fibrotic disease (45-47).

In addition to activation of TLR signaling, we show that pericytes activate the NLRP3 inflammasome in response to tissue injury and secrete major proinflammatory cytokines IL-1 and IL-18. Consistently with studies in liver hepatocytes (48), this report implicates pericytes as a significant source of inflammasomeactivated cytokines leading to recruitment of multiple leukocyte lineages. When MyD88 signaling was rendered deficient specifically in pericytes, we observed a 6-fold decrease in neutrophil recruitment and decreased numbers of activated proinflammatory macrophages in the kidney after ischemic injury.

We also show here that pericytes not only secrete and induce paracrine response to ILs but are also able to respond to IL-1 cytokines via a MyD88-dependent mechanism in an autocrine manner, thereby amplifying inflammation and potentially exacerbating fibrosis. Previous reports in ischemic kidney injury suggest that the blockade of IL-1 $\beta$ signaling by IL1RA has a very modest effect on kidney injury extent and progression to fibrosis (30). Our studies highlight that while IL-1 stimulates enhanced inflammatory signaling in pericytes, it has little direct impact on fibrogen- ic differentiation. By inhibiting IRAK4, which is downstream of many MyD88-dependent receptors, including IL-1 receptor (Figure 7), we have had a greater impact on the progressive responses to kidney injury than by simply blocking IL-1 signaling.

Our findings have important implications for the development of novel therapies for fibrosing disease, in which downstream effectors of MyD88 activation including IRAK1, IRAK4, and TRAF6 may be important novel targets (49). In this study we show that pharmacological inhibition of MyD88 signaling using a novel orally bioavailable small-molecule inhibitor of IRAK4 blocks human pericyte activation responses ex vivo, and can be safely administered to reduce MyD88 signaling in vivo, to benefit the tissue response to injury. Moreover, independent observations suggest that IRAK4 is a potential target for chronic inflammatory disease (30).

We conclude that pericytes possess unique properties and activate both inflammation and fibrogenesis in response to tissue injury via a TLR- and MyD88-dependent mechanism, leading to cytokine and chemokine production, recruitment of leukocytes, increased tissue damage, and extracellular matrix deposition, and pharmacological inhibition of this response dials down inflammatory and fibrotic responses while promoting regeneration.

\section{Methods}

Materials were from Sigma-Aldrich unless stated otherwise.

Pathway enrichment analysis. Gene profiling of human acute kidney injury compared with healthy kidneys, pericytes/fibroblasts, and other cell lineages from healthy and injured kidneys by TRAP was previously reported $(22,24,25)$ and data sets deposited to GEO DataSets. For enrichment analysis of biological process ontology, differentially expressed genes were analyzed in DAVID (23), and processes were selected based on $P$ less than 0.05 .

DAMP preparation. IRI and healthy kidney DAMPs were collected 24 hours after surgery under sterile conditions using methods previously described (26). Histone H4 (Millipore) was used at a final concentration of $10 \mu \mathrm{g} / \mathrm{ml}$, total histones (Worthington Biochemical) at a final concentration of $10 \mu \mathrm{g} / \mathrm{ml}$, and HMGB1 (Sigma-Aldrich) at a final concentration of $10 \mu \mathrm{g} / \mathrm{ml}$.

Pericyte purification and culture. Mouse pericytes were puri-

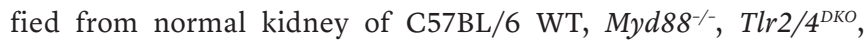

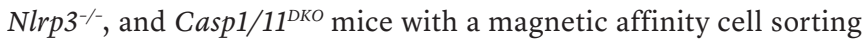
system (Miltenyi Biotec) as described previously (8). Human pericytes were purified from fetal human kidneys obtained following voluntary pregnancy interruptions (day 110 to day 130 of gestation) performed at the University of Washington Medical Center (IRB447773EA University of Washington) or through a sponsored research agreement with New England Medical Center as previously described (38). Informed consent for the use of fetal tissues was obtained from all patients. The single-cell preparation was depleted of epithelial cells by passing through an anti-CD326 and lotus lectin magnetic bead Column (Miltenyi Biotec). Pericytes were further sorted as a population positive for PDGFR $\beta(1.5 \mu \mathrm{g} / \mathrm{ml}$; BioLegend; catalog 323608) and negative for CD45 (1 $\mu \mathrm{g} / \mathrm{ml}$; BioLegend; catalog 304022) and CD31 (1:50; Pharmingen; catalog 555446). Pericytes were cultured in pericyte medium as previously described (8) on $0.2 \%$ gelatin-coated plates. Purity of pericyte cultures was confirmed by FACS to represent $>99 \%$ PDGFR $\beta^{+}$populations and 
be free of $\mathrm{CD} 45^{+}, \mathrm{CD} 326^{+}$, or $\mathrm{CD} 31^{+}$cells. All pericytes were studied at low passages (below passage 5 ).

Cell culture assays. Ultrapure LPS $(100 \mathrm{ng} / \mathrm{ml}), \mathrm{Pam}_{3} \mathrm{CSK}_{4}(300$ $\mathrm{ng} / \mathrm{ml})$, FSL1 (100 ng/ml), Poly(I:C) $(10 \mu \mathrm{g} / \mathrm{ml})$, flagellin (100 ng/ml), gardiquimod $(3 \mu \mathrm{g} / \mathrm{ml})$, and ODN1826 $(5 \mu \mathrm{M})$ were from Invivogen. ATP (5 mM) was from Sigma-Aldrich. IL-1 $\beta$ (10 ng/ml) and TGF- $\beta$ (10 $\mathrm{ng} / \mathrm{ml})$ were from PeproTech. Recombinant human IL1RA $(10 \mu \mathrm{g} /$ $\mathrm{ml}$ ) was from PeproTech (catalog 200-01RA). MyD88 inhibitor from Novus Biologicals (NBP2-29328) was used at concentrations of 10 and $100 \mu \mathrm{M}$. TGF- $\beta 1,2,3$ antibody, clone 1D11, was purchased from R\&D Systems and used at $10 \mu \mathrm{g} / \mathrm{ml}$. TGFßRI/II inhibitor LY2109761 from Selleckchem was used at $10 \mu \mathrm{M}$.

Pericytes (P3-P5) were cultured in 6-well gelatin-coated plates at a density of $4 \times 10^{5}$ cells $/ \mathrm{ml}$ for 24 hours in pericyte medium. Cells were then washed 5 times with PBS and cultured overnight in serum-free pericyte medium. TLR ligands at the above-stated final concentrations or $10 \%-20 \%$ kidney DAMPs in medium were used to activate cells. For transcriptional analysis, cells were washed in PBS, then lysed in Trizol or RLT buffer (Qiagen) at 3, 6, 16, 24, or 72 hours. For supernatant analysis, pericytes were cultured for 6 hours with activating factors, then washed 5 times with PBS before conditioning of full medium for 24 hours. Supernatants were harvested at 24 hours, centrifuged at 12,000 rpm to remove any particulate matter, and stored at $-20^{\circ} \mathrm{C}$ before analysis. To quantify IL- 1 cleavage by the inflammasome, pericytes were cultured in T75 flasks in $5 \mathrm{ml}$ of supernatant. After conditioning, the supernatant was concentrated 50 times using Pierce Protein Concentrators 9K MWCO (catalog 87748) according to the manufacturer's instructions. Migration was evaluated in a wound-healing scratch assay in 96-well plates using IncuCyte from Essen BioScience.

For gene silencing, specific siRNA or scrambled siRNA (Supplemental Table 4) from Thermo Fisher was transfected into pericytes at $40 \mathrm{pmol}$ using RNAiMax Lipofectamine transfection reagent from Life Technologies 48 hours before the experiment using methods previously described (50) and following protocols from the manufacturer.

Animals and animal studies. Foxd1 $1^{+/ C r e} M y d 88^{\text {flfl }}$ mice on the C57BL/6 genetic background were generated by crossing of female Foxd $1^{+/ C r e} M y d 88^{+/ f l}$ mice with male $M y d 88^{f / f l}$ mice. $M y d 88^{f / f l}$ mice were obtained from The Jackson Laboratory [CBy.129P2(B6)-Myd88tm1Defr/J]. Foxd1 ${ }^{+/ C r e}$ were generated as previously described (6). Six2-GFPCre and CSF1R-iCre were crossed with Myd88 $8^{f / f l}$ mice as previously described $(51,52)$ to bring about Myd88 locus recombination in kidney epithelium or monocytes and neutrophils, respectively. Experimental mice were identified by PCR to identify the floxed allele and the Cre insertion at the Foxd1 locus using primers as previously described (39). Col1a1-GFP ${ }^{T g}, \mathrm{Tlr}^{-{ }^{--}}, \mathrm{Tlr} 4^{-/}, \mathrm{Tlr} 2 / 4^{\mathrm{DKO}}$, Myd88 $8^{--}$, Nlrp3 $3^{--}$, and Casp1/11 ${ }^{\text {DKO }}$ were generated and genotyping performed as previously described $(8,37,53,54)$ and maintained on the C57BL/6 background. Kidney IRI studies were performed as previously described on mutant, littermate control, or C57BL/6J mice (55). Mice were euthanized at 24 hours or 5, 7, 14, or 16 days after surgery. In some experiments a nephrectomy of the contralateral kidney was performed 14 days after IRI as previously described (56). BIIB-IRAK4i (Biogen) in $250 \mu \mathrm{l}$ of vehicle (50\% PEG/citric acid) or an equal volume of vehicle alone was given by oral gavage at $75-100 \mathrm{mg} / \mathrm{kg}$ once daily to mice. All operators were blinded to the compound or genotype. All studies were performed under protocols approved by Institutional Animal Care and Use Committees at the University of Washington or Biogen.

Histology staining and evaluation. Kidneys were resected after systemic perfusion with ice-cold PBS. Paraffin-embedded sections of kidney fixed with neutral-buffered formalin were used for periodic acid-Schiff (PAS) and Sirius red staining. Epithelial injury was quantified in PAS-stained kidney sections as previously described (57). Interstitial fibrosis was quantified in Sirius red-stained sections as previously described (6).

Immunofluorescence staining. Mouse tissues were prepared and stained as previously described (58). For fluorescence detection, primary antibodies against the following proteins were used for immunolabeling: $\alpha$ SMA-Cy3 (1:200, clone 1A4; Sigma-Aldrich), F4/80 (1:300; Life Technologies), PDGFR $\beta$ (1:400; eBioscience), Ly6G (1:400; eBioscience), and CD169 (1:100; LifeSpan Biosciences; LS-6188785). Cytokines were detected in pericytes using anti-PDGFR $\beta$ antibodies to detect pericytes and the following primary antibodies to detect cytokines: anti-IL-1 $($ R\&D Systems; $5 \mu \mathrm{g} / \mathrm{ml}$ ), antiMCP-1 (R\&D Systems; $1 \mu \mathrm{g} / \mathrm{ml}$ ), and anti-IL-6 (BioLegend; $1 \mu \mathrm{g} / \mathrm{ml}$ ). Fluorescence-conjugated affinity-purified secondary antibody labeling (1:400-1:800; Jackson ImmunoResearch Laboratories), colabeling with DAPI, mounting with ProLong Gold/DAPI or VectaShield/ DAPI, and image capture and processing using confocal imaging were carried out as previously described (8). Detection of apoptotic cells was performed using the In Situ Cell Death TUNEL Detection Kit (Roche) on paraffin sections, and quantification was performed as previously described (50). Images were captured by confocal fluorescence microscopy (Zeiss LSM 710).

Western blotting. Kidneys were homogenized in ice-cold RIPA cell lysis buffer (Boston Bioproducts) including HALT protease and phosphatase inhibitor Cocktail (Thermo Fisher) with $1 \mathrm{mM}$ PMSF using the Omni Bead Ruptor Homogenizer. Lysates were centrifuged for 10 minutes at $14,000 \mathrm{~g}$, and the supernatant was taken for protein determination. Cell extracts containing $15 \mu \mathrm{g}$ of protein were prepared in SDS sample buffer and subjected to SDS-PAGE. Proteins were transferred onto nitrocellulose paper. After the transfer, immunostaining was performed as previously described in detail. Antibodies were diluted 1:1,000 in blocking buffer. Bands were detected by the enhanced chemiluminescence (ECL) method (Pierce) as recommended by the manufacturer and luminescence captured by ChemiDoc MP Imaging system (Bio-Rad). The following primary antibodies were used: anti-histone $\mathrm{H} 4$ (Abcam; 1:1,000), anti-HMGB1 (BioLegend; 1:500), $\beta$-actin (Santa Cruz Biotechnology; 1:10,000), anti-HMGB1 (BioLegend; 1:1,000), anti-IL-1 $\beta$ (R\&D Systems; 1:1,000), anti-NLRP3 (Fisher Scientific; 8N8E9, 1:1,000), anti-MyD88 (E-11; Santa Cruz Biotechnology; sc-74532, 1:1,000), and anti-TGF $\beta$ RI (S-165; Aviva Systems; OAA100768, 1:1,000).

Quantification of secreted proteins. Secreted proteins were collected in cell culture supernatants as previously described (26). Secreted proteins were measured by ELISA (IL-6, MCP-1, IL-1 $\beta$, and IL-18) (R\&D Systems or BioLegend) or by 20-plex cytokine magnetic bead array (Invitrogen) following the manufacturer's instructions.

Quantitative RT-PCR. Total RNA was extracted using an RNeasy Plus Mini Kit (Qiagen). Purity was determined by the A260/A280 ratio. cDNA was synthesized using oligo-dT and random primers 
(iScript Reverse Transcription Supermix; Bio-Rad). Quantitative PCR (qPCR) was performed using QuantStudio 7 Flex Real-Time PCR System (Life Technologies) and TaqMan Gene Expression Assays according to the manufacturer's instructions (Life Technologies). The specific TaqMan probe sets used in qPCR are listed in Supplemental Table 3 (Life Technologies). All values were normalized to Gapdh or GAPDH and graphed as fold change versus control.

Phosphorylation assay. The phosphorylation assay was carried out using an AlphaScreen ${ }^{\mathrm{R}}$ kit from PerkinElmer according to the manufacturer's protocol with $1 \mathrm{nM}$ IRAK $4,1.6 \mu \mathrm{M}$ peptide, $1 \mathrm{mM}$ ATP in reaction buffer ( $50 \mathrm{mM}$ HEPES, $60 \mathrm{mM} \mathrm{NaCl}, 5 \mathrm{mM} \mathrm{MgCl}{ }_{2}$, $0.25 \mathrm{mM} \mathrm{MnCl}_{2}, 2 \mathrm{mM}$ DTT, 0.01\% BSA, 0.01\% Tween-20) for 3.5 hours at room temperature.

Binding assays (Discover $X$ ). Kinase-tagged T7 phage strains were grown in parallel in 24-well blocks in an E. coli host derived from the BL21 strain. E. coli were grown to log phase, infected with T7 phage from a frozen stock $(\mathrm{MOI}=0.4)$, and incubated with shaking at $32^{\circ} \mathrm{C}$ until lysis (90-150 minutes). The lysates were centrifuged $(6,000 \mathrm{~g})$ and filtered $(0.2 \mu \mathrm{m})$ to remove cell debris. The remaining kinases were produced in HEK-293 cells and subsequently tagged with DNA for qPCR detection. Streptavidin-coated magnetic beads were treated with biotinylated small-molecule ligands for 30 minutes at room temperature to generate affinity resins for kinase assays. The liganded beads were blocked with excess biotin and washed with blocking buffer (SeaBlock [Pierce], 1 \% BSA, 0.05\% Tween-20, 1 mM DTT) to remove unbound ligand and to reduce nonspecific phage binding. Binding reactions were assembled by combining of kinases, liganded affinity beads, and test compounds in $1^{\prime}$ binding buffer (20\% SeaBlock, 0.17' PBS, 0.05\% Tween-20, 6 mM DTT). Test compounds were prepared as $40^{\prime}$ stocks in $100 \%$ DMSO and directly diluted into the assay. All reactions were performed in polypropylene 384-well plates in a final volume of $0.04 \mathrm{ml}$. The assay plates were incubated at room temperature with shaking for 1 hour, and the affinity beads were washed with wash buffer (1' PBS, 0.05\% Tween-20). The beads were then resuspended in elution buffer ( $1^{\prime}$ PBS, 0.05\% Tween-20, $0.5 \mu \mathrm{M}$ nonbiotinylated affinity ligand) and incubated at room temperature with shaking for 30 minutes. The kinase concentration in the eluates was measured by qPCR.
Statistics. Data are presented as the means \pm SEM. Statistically significant differences between groups were determined by 2-tailed Student's $t$ test, 1-way ANOVA, or 2-way ANOVA with Bonferroni correction for multiple comparisons with a $95 \%$ CI. A $P$ value of 0.05 or lower was considered significant.

Study approval. Human studies were approved through University of Washington Medical Center (IRB447773EA University of Washington) or through a sponsored research agreement with New England Medical Center. All animal studies were performed under protocols approved by Institutional Animal Care and Use Committees at the University of Washington or Biogen.

\section{Author contributions}

All authors performed experiments; all authors, including BGJ and $\mathrm{KM}$, analyzed data; IAL, SN, KMG, WAA, and JSD analyzed and interpreted results of the experiments; IAL, SN, JJC, KMG, and JSD prepared figures; JSD, IAL, and KMG edited and revised the manuscript; all authors approved the final version of the manuscript; and IAL, KMG, IGG, and JSD conceived and designed research.

\section{Acknowledgments}

These studies were funded by Biogen, by NIH grants (DK087389, DK093493, DK094768, HL122895, TR000504), and by an American Heart Association grant (12040023). We thank Chris Roberts, Raghavendra Hosur, Dania Rabah, Holly Legault, Kalyan Vasudevan, Tracy Jenkins, Timothy Chan, Matthew Flegal, David Koske, Peter Juhasz, Graham Marsh, Luke Barron, Shuyu Ren, and Angela Huang (Biogen); Kimberly Muczinski, Julia Lichtnekert, Anoria Haick, and Alan Aderem (University of Washington); and Bill Stallcup (Burnham Institute) for assistance.

Address correspondence to: Irina A. Leaf, Biogen, Building 6, 115 Broadway, Cambridge, Massachusetts 02142, USA. Phone: 617.914.4137; E-mail: irina.leaf@biogen.com. Or to: Jeremy S. Duffield, UW Medicine, 850 Republican Street, Box 358052, Seattle, Washington 98109, USA. Phone: 206.685.8456; E-mail: jeremysd@u.washington.edu.
1. Duffield JS. Cellular and molecular mechanisms in kidney fibrosis. JClin Invest. 2014;124(6):2299-2306.

2. Zeisberg M, Kalluri R. Fibroblasts emerge via epithelial-mesenchymal transition in chronic kidney fibrosis. Front Biosci. 2008;13:6991-6998.

3. LeBleu VS, et al. Origin and function of myofibroblasts in kidney fibrosis. Nat Med. 2013;19(8):1047-1053.

4. Cieslik KA, Trial J, Entman ML. Mesenchymal stem cell-derived inflammatory fibroblasts promote monocyte transition into myeloid fibroblasts via an IL-6-dependent mechanism in the aging mouse heart. FASEB J. 2015;29(8):3160-3170.

5 . Asada N, et al. Dysfunction of fibroblasts of extrarenal origin underlies renal fibrosis and renal anemia in mice. J Clin Invest. 2011;121(10):3981-3990.

6. Humphreys BD, et al. Fate tracing reveals the pericyte and not epithelial origin of myofibroblasts in kidney fibrosis. Am J Pathol. 2010;176(1):85-97.
7. Kramann R, et al. Perivascular $\mathrm{Gli1}^{+}$progenitors are key contributors to injury-induced organ fibrosis. Cell Stem Cell. 2015;16(1):51-66.

8. Lin SL, Kisseleva T, Brenner DA, Duffield JS. Pericytes and perivascular fibroblasts are the primary source of collagen-producing cells in obstructive fibrosis of the kidney. Am J Pathol. 2008;173(6):1617-1627.

9. Alon R, Nourshargh S. Learning in motion: pericytes instruct migrating innate leukocytes. Nat Immunol. 2013;14(1):14-15.

10. Proebstl D, et al. Pericytes support neutrophil subendothelial cell crawling and breaching of venular walls in vivo. J Exp Med. 2012;209(6):1219-1234.

11. Armulik A, Genové G, Betsholtz C. Pericytes: developmental, physiological, and pathological perspectives, problems, and promises. Dev Cell. 2011;21(2):193-215.

12. Cooke VG, et al. Pericyte depletion results in hypoxia-associated epithelial-to-mesenchymal transition and metastasis mediated by met signaling pathway. Cancer Cell. 2012;21(1):66-81.

13. Meng MB, et al. Pericytes: a double-edged sword in cancer therapy. Future Oncol. 2015;11(1):169-179.

14. Pfister F, Przybyt E, Harmsen MC, Hammes HP. Pericytes in the eye. Pflugers Arch. 2013;465(6):789-796.

15. Rowley JE, Johnson JR. Pericytes in chronic lung disease. Int Arch Allergy Immunol. 2014;164(3):178-188.

16. Weiner GM, Ducruet AF. Preventing ischemia after stroke: are pericytes the answer? Neurosurgery. $2014 ; 75(2)$ :N21-N23.

17. Winkler EA, Sagare AP, Zlokovic BV. The pericyte: a forgotten cell type with important implications for Alzheimer's disease? Brain Pathol. 2014;24(4):371-386.

18. Massagué J. TGF- $\beta$ signaling in development and disease. FEBS Lett. 2012;586(14):1833.

19. Rock KL, Latz E, Ontiveros F, Kono H. The sterile inflammatory response. Annu Rev Immunol. 
2010;28:321-342.

20. Bergsbaken T, Fink SL, Cookson BT. Pyroptosis: host cell death and inflammation. Nat Rev Microbiol. 2009;7(2):99-109.

21. Dinarello CA. Immunological and inflammatory functions of the interleukin-1 family. Annu Rev Immunol. 2009;27:519-550.

22. Famulski KS, et al. Molecular phenotypes of acute kidney injury in kidney transplants. J Am Soc Nephrol. 2012;23(5):948-958.

23. Dennis G, et al. DAVID: Database for Annotation, Visualization, and Integrated Discovery. Genome Biol. 2003;4(5):P3.

24. Grgic I, et al. Translational profiles of medullary myofibroblasts during kidney fibrosis. J Am Soc Nephrol. 2014;25(9):1979-1990.

25. Liu J, et al. Cell-specific translational profiling in acute kidney injury. J Clin Invest. 2014;124(3):1242-1254.

26. Campanholle G, et al. TLR-2/TLR-4 TREM-1 signaling pathway is dispensable in inflammatory myeloid cells during sterile kidney injury. PLoS One. 2013;8(7):e68640.

27. Allam R, et al. Histones from dying renal cells aggravate kidney injury via TLR2 and TLR4. J Am Soc Nephrol. 2012;23(8):1375-1388.

28. Yu M, et al. HMGB1 signals through tolllike receptor (TLR) 4 and TLR2. Shock. 2006;26(2):174-179.

29. Ozaki E, Campbell M, Doyle SL. Targeting the NLRP3 inflammasome in chronic inflammatory diseases: current perspectives. J Inflamm Res. 2015;8:15-27.

30. Haq M, Norman J, Saba SR, Ramirez G, Rabb H. Role of IL-1 in renal ischemic reperfusion injury. JAm Soc Nephrol. 1998;9(4):614-619.

31. Zhang JD, et al. Type 1 angiotensin receptors on macrophages ameliorate IL-1 receptor-mediated kidney fibrosis. J Clin Invest. 2014;124(5):2198-2203.

32. Burns K, et al. MyD88, an adapter protein involved in interleukin-1 signaling. J Biol Chem. 1998;273(20):12203-12209.

33. Schrimpf C, Duffield JS. Mechanisms of fibrosis: the role of the pericyte. Curr Opin Nephrol Hypertens. 2011;20(3):297-305.

34. Pulskens WP, et al. TLR4 promotes fibrosis but attenuates tubular damage in progressive renal injury. J Am Soc Nephrol. 2010;21(8):1299-1308.

35. Freudlsperger $C$, et al. TGF- $\beta$ and NF- $\kappa$ B signal pathway cross-talk is mediated through TAK1 and SMAD7 in a subset of head and neck cancers. Oncogene. 2013;32(12):1549-1559.

36. Feng XH, Derynck R. Ligand-independent activation of transforming growth factor (TGF) beta signaling pathways by heteromeric cytoplasmic domains of TGF- $\beta$ receptors. J Biol Chem. 1996;271(22):13123-13129.

37. Chun CD, Liles WC, Frevert CW, Glenny RW, Altemeier WA. Mechanical ventilation modulates Toll-like receptor-3-induced lung inflammation via a MyD88-dependent, TLR4independent pathway: a controlled animal study. BMC Pulm Med. 2010;10:57.

38. Gomez IG, et al. Anti-microRNA-21 oligonucleotides prevent Alport nephropathy progression by stimulating metabolic pathways. J Clin Invest. 2015;125(1):141-156.

39. Nakagawa N, et al. Dicer1 activity in the stromal compartment regulates nephron differentiation and vascular patterning during mammalian kidney organogenesis. Kidney Int. 2015;87(6):1125-1140.

40. Harberts E, Fishelevich R, Liu J, Atamas SP, Gaspari AA. MyD88 mediates the decision to die by apoptosis or necroptosis after UV irradiation. Innate Immun. 2014;20(5):529-539.

41. Burns K, Janssens S, Brissoni B, Olivos N, Beyaert $\mathrm{R}$, Tschopp J. Inhibition of interleukin 1 receptor/Toll-like receptor signaling through the alternatively spliced, short form of MyD88 is due to its failure to recruit IRAK-4. J Exp Med. 2003;197(2):263-268.

42. Armulik A, et al. Pericytes regulate the bloodbrain barrier. Nature. 2010;468(7323):557-561.

43. Betsholtz C, Armulik A. Homeostatic functions of vascular endothelial growth factor in adult microvasculature. Am J Physiol Heart Circ Physiol. 2006;290(2):H509-H511.

44. Guo J, Friedman SL. Toll-like receptor 4 signaling in liver injury and hepatic fibrogenesis. Fibrogenesis Tissue Repair. 2010;3:21.

45. Wetmore JB, Hung AM, Lovett DH, Sen S, Quershy O, Johansen KL. Interleukin-1 gene cluster polymorphisms predict risk of ESRD. Kidney
Int. 2005;68(1):278-284.

46. Yoshida T, et al. Association of genetic variants with chronic kidney disease in Japanese individuals with type 2 diabetes mellitus. Int J Mol Med. 2009;23(4):529-537.

47. Krüger B, et al. Donor Toll-like receptor 4 contributes to ischemia and reperfusion injury following human kidney transplantation. Proc Natl Acad Sci U S A. 2009;106(9):3390-3395.

48. Wree A, et al. NLRP3 inflammasome activation results in hepatocyte pyroptosis, liver inflammation, and fibrosis in mice. Hepatology. 2014;59(3):898-910.

49. Li X. IRAK4 in TLR/IL-1R signaling: possible clinical applications. Eur J Immunol. 2008;38(3):614-618.

50. Schrimpf C, et al. Pericyte TIMP3 and ADAMTS1 modulate vascular stability after kidney injury. JAm Soc Nephrol. 2012;23(5):868-883.

51. Lin SL, et al. Macrophage Wnt7b is critical for kidney repair and regeneration. Proc Natl Acad Sci US A. 2010;107(9):4194-4199.

52. Kawakami T, et al. Deficient autophagy results in mitochondrial dysfunction and FSGS. JAm Soc Nephrol. 2015;26(5):1040-1052.

53. Kuida K, et al. Altered cytokine export and apoptosis in mice deficient in interleukin- $1 \beta$ converting enzyme. Science. 1995;267(5206):2000-2003.

54. Martinon F, Pétrilli V, Mayor A, Tardivel A, Tschopp J. Gout-associated uric acid crystals activate the NALP3 inflammasome. Nature. 2006;440(7081):237-241.

55. Duffield JS, et al. Restoration of tubular epithelial cells during repair of the postischemic kidney occurs independently of bone marrow-derived stem cells. JClin Invest. 2005;115(7):1743-1755.

56. Chau BN, et al. MicroRNA-21 promotes fibrosis of the kidney by silencing metabolic pathways. Sci Transl Med. 2012;4(121):121ra18.

57. Ren S, et al. LRP-6 is a coreceptor for multiple fibrogenic signaling pathways in pericytes and myofibroblasts that are inhibited by DKK-1. Proc Natl Acad Sci U S A. 2013;110(4):1440-1445.

58. Campanholle G, Ligresti G, Gharib SA, Duffield JS. Cellular mechanisms of tissue fibrosis. 3 . Novel mechanisms of kidney fibrosis. Am J Physiol Cell Physiol. 2013;304(7):C591-C603. 